\section{Introduction: Special Issue on the Ethics of Incentives in Healthcare}

\author{
Anca Gheaus, ${ }^{1}$ Verina Wild ${ }^{2}$
}

This special issue is the result of a conference organised by Verina Wild and Anca Gheaus, at the Ludwig-Maximilians University of Munich in December 2014. The conference addressed normative issues raised by the use of incentive mechanisms to promote better health, and included papers by most contributors to this special issue. So far, the normative discussion on health incentives focussed on questions of autonomy, paternalism, motivation and responsibility. This resulting special issue responds to a need to expand the normative analysis of such measures to other issues of justice, which have so far been largely ignored.

Recent policies and programmes in health prevention tend to appeal to, and encourage, individual responsibility with respect to lifestyle choices. One way of advancing this goal is via schemes that provide individuals with incentives to live healthy lives. For example, individuals may be offered discounted health insurance rates if they adopt healthy lifestyles or be given vouchers to purchase healthy food or to use fitness centres. Such programmes often use so-called 'nudging' mechanisms, meant to motivate people without coercively interfering with their private choices and to improve health outcomes without overregulating the market for products that are detrimental to health. They also raise many normative questions.

Health incentive programmes put pressure on us to rethink how to balance reasons of freedom, solidarity and justice in the design of public health policies. Angus Dawson's article is a criticism to the use of the ladder metaphor for interventions in healthcare. The Nuffield Council of Bioethics first introduced this influential metaphor in its 2007 publication Public Health: Ethical Issues, a piece on normative issues and policy design in public health. This publication recommends that we promote healthier behaviours according to an 'intervention ladder' in which the less liberty-restricting policies are seen

${ }^{1}$ Universitat Pompeu Fabra, Barcelona, Spain ${ }^{2}$ Department of Philosophy, Ludwig-Maximilians University of Munich, Munich, Germany

Correspondence to Dr Anca Gheaus, Universitat Pompeu Fabra, C/Ramon Trias Fargas 25-27, Barcelona 08005, Spain; agheaus@gmail.com as more desirable. As Dawson notes, it is unclear how other values-such as fairness in the distribution of health resources, effectiveness and precaution-are to be weighed against freedom in this context. Moreover, the ladder metaphor makes it harder to see that these other values generate normative considerations that are as important as, or more important than, liberty. Dawson emphasises the importance of these other values and recommends that we look elsewhere to find better policy tools. Policies designed to promote health may also result in (possibly undesirable) value changes in society. Sometimes people object to the use of financial incentives in the promotion of healthier behaviours by saying that people's health-related choices ought to be guided by non-financial values. Rebecca Brown offers a critical analysis of the claim, famously defended by Michael Sandel, that monetary incentives corrupt values that ordinarily regulate exchange and behaviour in previously nonmonetised contexts. Brown argues that Sandel's claim does not necessarily apply to health incentive schemes. At the moment, we lack evidence that such schemes are either inefficient or corrosive of ethically desirable attitudes. More investigation is certainly needed in the likely effects of these schemes on public values. At least some incentive programmes can balance well all the values at stake. Justin Healy and his co-authors look at policies that create financial incentives for adherence to antiretroviral treatment for adolescents in sub-Saharan Africa. They show that, based on existing evidence, we need not worry that either distributive justice, or liberty, or ethically sound motivation would be compromised by the implementation of such policies. However, more empirical research is needed.

Of particular interest is the question of how of health incentive programmes impact on justice, and a majority of the articles are interested in their potentially inequality-enhancing effect. Verina Wild and Bridget Pratt are interested in potential long-term harms for systematically disadvantaged groups resulting from research on health incentive programmes. They argue that the initiators of research bear the responsibility of protecting the most vulnerable from significant risk of such harm and propose strategies of harm mitigation. They recommend the inclusion of incentives that are accessible for underprivileged groups, the monitoring of the incentives' effects on health disparities and the publication of any conclusions on how the worst off are negatively affected for the benefit of future researchers.

The design of particular programmes seems crucial in relation to equality. Kalle Grill notes that schemes aiming to produce healthier behaviours by changing the costs associated with relevant options are successful only if individuals in the target population can respond rationally to such incentives. In fact, some do and some don't, and those who are less capable of prudent choices tend to be among the least advantaged in society: the ability to make rational choices affects one's well-being in general. This means that health incentive schemes can aggravate inequalities by benefiting more the relatively better off. In contrast, the success of schemes relying on the restrictions of physically available options, and on making some options more salient, does not depend on how rational the individuals in the target population are. Grill concludes that this is an equity-based reason to favour the latter schemes over schemes that rely on cost-affecting incentives. A similar concern-namely that health-promoting programmes can increase inequalities in the well-being of their target population-is explored by Kristin Voigt. Her own focus is on people's different capacities to respond to the programmes due to background inequalities in income and/or to the existence of previous health conditions which may hinder some individuals' ability to benefit. Like Grill, Voigt is interested in how we may design incentive mechanisms to avoid this danger and offers some practical suggestions. In the same spirit, Carleigh Krubiner and Maria Meritt emphasise that conditional cash-transfer programmes are unlikely to benefit everybody equally. They discuss several considerations that ought to be balanced in designing programmes, such as efficiency, risk reduction, feasibility and externalities. On Krubiner's and Meritt's account, distributive issues-that is, the reduction in inequities and the promotion of wellbeing for the least well off-have to be of primary, but not exclusive, concern.

This JME special issue also discusses the question of what various theories of justice can contribute to a better analysis of health incentive programmes. Luck egalitarians have long been charged with an inability to explain why we have duties of 
justice to assist people who have voluntarily chosen to jeopardise their own needs, and who could have avoided to do so at little cost to themselves. Anca Gheaus argues that luck egalitarianism can indeed explain why such duties exist: meeting people's basic needs is necessary to preserve solidarity. Solidarity has both instrumental and non-instrumental value in virtue of making people who live in solidary societies better off. Yet, solidarity is akin to a public good in that it cannot be provided only to some, but not to other, members of a society. Therefore, there is a duty of justice owed to prudent citizens to assist even the non-prudent citizens in the satisfaction of their basic needs to preserve solidarity for all.

Funding The research leading to this special issue has partly received funding from the European Union's Seventh Framework Programme FP7/2007-2013 under Grant Agreement no. 602386, Credits4Health.

Competing interests None declared.

Provenance and peer review Commissioned internally peer reviewed.
To cite Gheaus A, Wild V. J Med Ethics 2017;43:138-139.

Accepted 29 December 2016 